\title{
Correlation between Changes in Leg Blood Flow and Ankle-Brachial Pressure Index: A Study Using Laser Doppler Flowmeter - The 1st Report-
}

\author{
Kazuyoshi Suzuki, PT, ${ }^{1,2}$ Miho Sekiguchi, MD, PhD, ${ }^{2}$ Hirofumi Midorikawa, MD, PhD, ${ }^{3}$ Koichi Sato, MD, PhD, \\ Kazuyoshi Akase, ${ }^{5}$ Renshi Sawada, D.Eng, ${ }^{5}$ and Shin-ichi Konno, MD, $\mathrm{PhD}^{2}$
}

Objective: The objective of this study was to use non-invasive laser Doppler flowmeter to measure changes in blood flow in peripheral vessels in the legs before and after stress induced by leg elevation stress test and investigate correlations with the ankle-brachial pressure index (ABI).

Methods: Subjects included 28 patients over 20 years of age (mean, 73 years) who reported chiefly of leg symptoms such as intermittent claudication, numbness, chills, or cramps had been examined at the study institution, and agreed to participate in the study. The ABI of both legs was measured, and patients were divided into two groups: low ABI (ABI $\leq 0.9)$ and normal ABI (ABI $\geq 0.9)$. Blood flow in the big toe was measured using a box-type laser Doppler flowmeter before, during, and after leg-elevation stress. Amplitude of the recorded waveform and changes in blood flow were compared.

Results: Average ABI was $1.09 \pm 0.10$ in the normal ABI group (33 legs) and $0.68 \pm 0.17$ in the low ABI group (21 legs). Amplitude before and during stress was significantly smaller in the low ABI group than in the normal ABI group $(p<0.01)$, and there was a significant correlation with ABI before and during stresses $(r=0.4606, r=0.5048$, respectively; $p<0.05)$. Change in blood flow during stress was significantly lower in the low ABI group than in the normal ABI group $(p<0.05)$. There was a significant correlation between change in blood flow during stress and ABI in both groups $(r=0.5073 ; p<0.05)$. There was also a significant correlation between change in blood flow and change in amplitude in both groups $(r=0.5477$; $p<0.05)$.

Conclusion: Results of this study show, that comparing amplitude and change in blood flow before and after leg extension and elevation stress, there was a correlation between change in blood flow and amplitude, and ABI during stress. A box-type laser Doppler flowmeter may provide a means of screening for peripheral arterial disease.

Key words: peripheral arterial disease, ankle-brachial pressure index, laser Doppler flowmeter, intermittent claudication, lumbar spinal stenosis

${ }^{1}$ Division of Rehabilitation, Iwase General Hospital, Sukagawa, Fukushima, Japan

${ }^{2}$ Department of Orthopedic Surgery, Fukushima Medical University School of Medicine, Fukushima, Fukushima, Japan

${ }^{3}$ Division of Cardiac and Vascular Surgery, Southern tohoku Research Institute for Neuroscience, Southern tohoku General Hospital, Koriyama, Fukushima, Japan

${ }^{4}$ Department of Cardiovascular Surgery, Sukagawa Hospital, Sukagawa, Fukushima, Japan

${ }^{5}$ Department of Intelligent Machinery and Systems, Graduate School of Engineering, Graduate School of Systems Life Sci- ences, Microsystems and Bioengineering Lab, Kyushu University, Fukuoka, Fukuoka, Japan

Received: July 20, 2010; Accepted: March 9, 2011

Corresponding author: Miho Sekiguchi MD, PhD. Department of Orthopedic Surgery, Fukushima Medical University School of Medicine, 1-Hikarigaoka, Fukushima, Fukushima 960-1295, Japan

Fax: +81-24-548-5505; Tel: +81-24-547-1276

E-mail: miho-s@fmu.ac.jp 


\section{INTRODUCTION}

$\mathrm{W}$ ith the increasing aging of society, the incidence of arteriosclerosis-related disorders such as hypertension, and cardiovascular disease is rising. Peripheral arterial disease (PAD) and lumbar spinal stenosis (LSS) sometimes have similar symptoms and commonly occur around the same age; thus it is important to distinguish these underlying conditions. ${ }^{1,2)}$ The overall prevalence of PAD is $3 \%-10 \%$, rising to $15 \%-20 \%$ over the age of 70 years. $^{3-5)}$ The presence of arterial lesions in the legs reflects arterial lesions throughout the body, and the survival rate is poor. ${ }^{6}$ It follows that PAD with intermittent vascular claudication requires early diagnosis and referral to a vascular specialist for early treatment. Measurement of the ankle-brachial pressure index (ABI) is an effective method of screening for PAD. ${ }^{7,8)}$ Pulse volume recording, toe brachial pressure index (TBI), Duplex ultrasonography, and magnetic resonance angiography are non-invasive methods for diagnosis of PAD.7) ABI does not show blood flow of the leg tissue directly. Instead, $\mathrm{ABI}$ is determined using laser Doppler flowmeter (LDF) along with blood pressure measurements." ${ }^{90)}$ However, conventional LDF can only be used at rest and with patients maintaining the same position. If a simple, non-invasive method to measure blood flow in the leg were available that allowed for continual leg movement, it would enable screening for vascular lesions. A micro wireless flowmeter integrated laser Doppler blood flowmeter (MILDF) has been developed. ${ }^{11-13)}$ MILDF provides similar results to measurement of blood flow with conventional LDF. In addition, MILDF is wireless, portable, and allows monitoring of diurnal blood flow variation. The objective of this study was to use MILDF to measure changes in blood flow in the peripheral arteries of the leg before and after stress imposed by leg elevation stress test and to investigate their correlation with $\mathrm{ABI}$.

\section{SubJects ANd Methods}

Subjects were 28 patients over 20 years of age who had been examined at the study institution due to report chiefly related to leg symptoms such as intermittent claudication, numbness, chills, or cramps, and who provided informed consent to participate in the study. Subjects included 26 men and 2 women, with a mean age of 73 years (range, 57-91; most were in their 70s). This study was approved by the ethics committee of the facilities concerned.
ABI values were measured for both legs of the subjects. Subjects lay supine on a bed with blood pressure cuffs fitted to their upper arms and ankles on both sides, and their blood pressure was measured using a VaSera VS-1000 (Fukuda Denshi, Japan). Then, blood flow in both great toes was measured. The MILDF ${ }^{1-13)}$ used in this study was a box type that measured $5.6 \mathrm{~mm} \times 12.0$ $\mathrm{mm} \times 17.5 \mathrm{~mm}$ and weighed $3 \mathrm{~g}$ (Fig. 1). This is approximately $1 / 300$ the size of conventional LDF. Blood flow signals detected by the sensor were transmitted to the main unit, where measurement values were displayed. The main unit was also a signal transmitter, capable of sending data directly to a computer by Bluetooth operating on an industrial, science, and medical (ISM) 2.4$2.485 \mathrm{GHz}$ wireless band. The blood flow waveform was continuously displayed on the computer screen and recorded from the data sent to the computer. As conventional LDF uses optical fibers for the input-output of laser light, movements of the optical fiber can result in noise. In this study, the fact that MILDF was wireless meant noise could be minimized. In addition, the miniaturized main unit also has the advantages of portability as well as the capability for continuous measurement and measurement during movement. It provides similar results to those of conventional $\mathrm{LDF}^{11-13)}$ Measurements were performed in the outcome patient room at room temperature of $18^{\circ} \mathrm{C}$. Only the subject and two observers were in the room during measurements.

The blood flow sensor was directly attached to the skin on one side of the great toe by double-sided tape. Subjects lay in the supine position, and blood flow measurements were started after subjects had spent at least 5 min resting on the bed. The blood flow waveform was checked on the computer screen, and blood flow was continuously recorded before, during, and after stress, as follows. Blood flow was measured for 60 seconds (s) in a resting state (before stress). Next, leg extension and elevation stress was imposed for $60 \mathrm{~s}$, and blood flow was measured while the stress was imposed (during stress). The leg extension and elevation stress comprised elevating the leg in an extended position at an angle of $60^{\circ}$ from the bed. ${ }^{14)}$ The leg was then returned to the bed, and blood flow was measured for $60 \mathrm{~s}$ after the stress had finished (after stress). Blood flow in the big toe of the opposite leg was also measured in the same way.

Blood flow waveform amplitude and change in blood flow were measured from the results obtained. Amplitude reflected each pulse beat. Amplitude was calculated from the waveform during each 60 -s period before, 


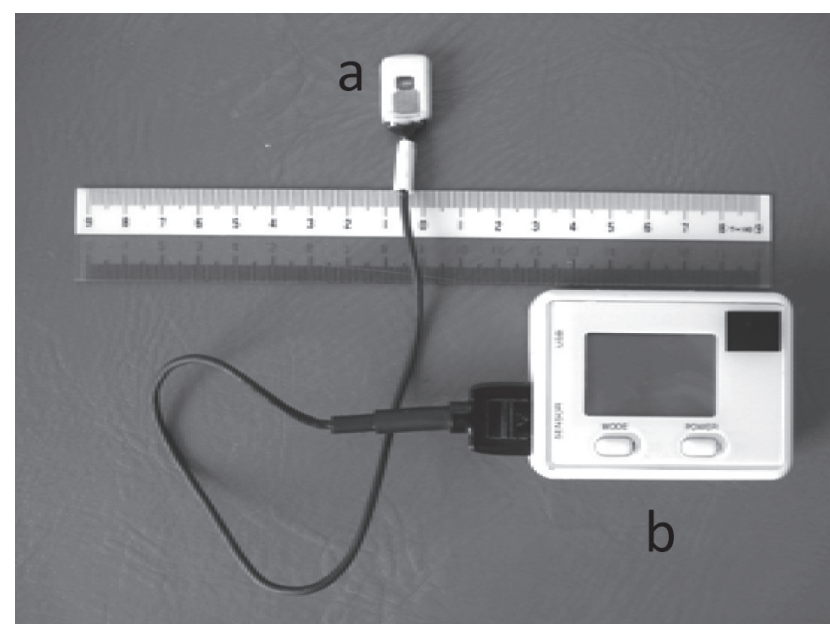

Fig. 1 Laser Doppler flowmeter.

(a) Box-type blood flow sensor and (b) portable flowmeter main unit.

during, and after stress by using 50-s periods excluding data from the $5 \mathrm{~s}$ before and after when the signal was affected by movement. Maximum and minimum amplitudes were selected from 10 points at 5-s intervals within the 50-s measurement periods, and the difference between them was taken as the amplitude size. Averages and standard deviations were calculated for the amplitudes at these 10 points before, during, and after stress, respectively. Change in amplitude was calculated by taking the amplitude before stress as $100 \%$ and calculating the change in average amplitude during and after stress, with amplitude before stress as the standard. Change in blood flow was calculated by using the waveform during 50 -s intervals, excluding the $5 \mathrm{~s}$ before and after when the signal was affected by movement. The average of 2500 blood flow plots during these $50 \mathrm{~s}$ was calculated. Change in blood flow was calculated by taking blood flow before stress as $100 \%$ and calculating the averages and standard deviations of the changes in blood flow during and after stress.

Analysis was performed on two designated groups: one with normal $\mathrm{ABI}(\mathrm{ABI}>0.9)$ and the other with low ABI (ABI $\leq 0.9){ }^{7)}$ Amplitude size and change in blood flow were compared between the two groups using a t-test. A $p$ value less than 0.05 was considered significant. The significance level was set at a risk factor $<5 \%$. Pearson's product-moment correlation coefficient was used to investigate correlations between ABI, amplitude size, and change in blood flow. A $p$ value of less than 0.05 was considered significant.

\section{RESULTS}

Analysis was performed on 54 of 56 legs in which blood flow was measured, excluding 2 legs for which the recorded waveform could not be analyzed. The normal ABI group comprised 33 legs, with an average ABI of $1.09 \pm 0.10$. The low ABI group comprised 21 legs, with an average $\mathrm{ABI}$ of $0.68 \pm 0.17$.

\section{Blood flow waveform characteristics and amplitude size}

Blood flow waveforms were characterized by a large amplitude in the normal ABI group and by a small amplitude in the low ABI group (Fig. 2). Amplitude before stress was $5.7 \pm 2.8 \mathrm{ml}(\mathrm{min} 100 \mathrm{~g})$ in the normal $\mathrm{ABI}$ group and $3.6 \pm 2.5 \mathrm{ml}(\mathrm{min} 100 \mathrm{~g})$ in the low ABI group. Amplitude was significantly smaller in the low ABI group than the normal ABI group $(p<0.01)$. There was a correlation between amplitude before stress and ABI $(r=0.4606 ; p<0.05)($ Fig. 3a).

\section{Change in amplitude during stress}

The change in amplitude during stress was $73.9 \% \pm$ $62.2 \%$ in the low ABI group and $108.7 \% \pm 42.6 \%$ in the normal ABI group. The amplitude was significantly decreased in the low ABI group compared with the normal ABI group $(p<0.05)$. The change in amplitude after stress was $100.9 \% \pm 19.4 \%$ in the low ABI group and $104.5 \% \pm 23.8 \%$ in the normal ABI group. There was no significant difference between the two groups. There was a correlation between change in amplitude during stress and ABI $(r=0.5048 ; p<0.05)$ (Fig. 3b).

\section{Change in blood flow during stress}

The change in blood flow during stress was $44.5 \% \pm$ $23.2 \%$ in the low $\mathrm{ABI}$ group and $66.0 \pm 18.5 \%$ in the normal ABI group. The blood flow was significantly decreased in the low ABI group compared with the normal ABI group $(p<0.05)$. The change in blood flow after stress was $116.2 \% \pm 30.2 \%$ in the low ABI group and $105.3 \% \pm 22.3 \%$ in the normal ABI group. There was no significant difference in change in blood flow between the two groups. There was a correlation between change in blood flow during stress and ABI $(r=0.5073 ; p<0.05)$ (Fig. 4).

When the cut-off point of the blood flow change was defined as $60 \%$, the flowmeter had a sensitivity of $71 \%$ and specificity of $58 \%$. When cut-off point was $65 \%$, the flowmeter had a sensitivity of $81 \%$ and a specificity of $55 \%$. 


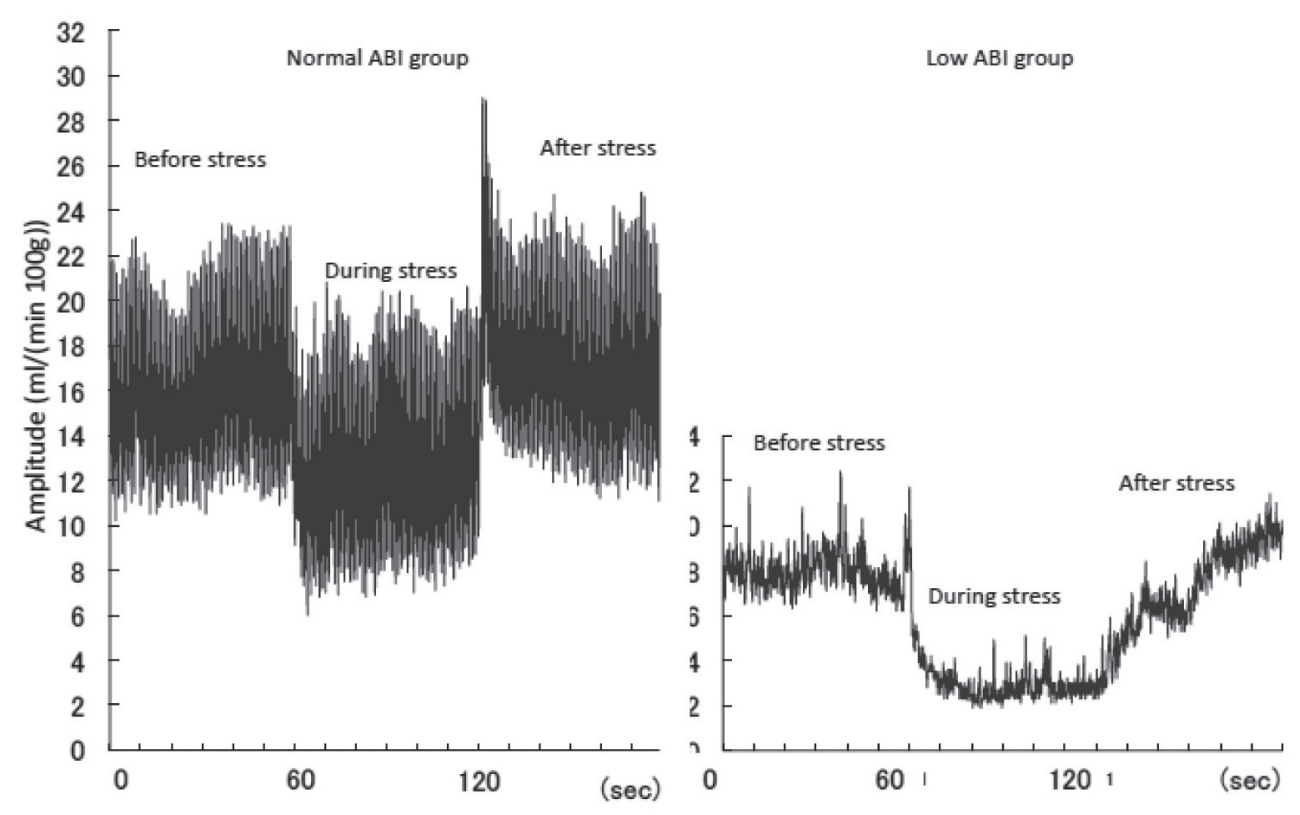

Fig. 2 Blood flow waveform characteristics.

The blood flow waveform in the normal ABI group was of large amplitude, whereas the amplitude was small in the low ABI group.

$\mathrm{ABI}$, ankle-brachial pressure index

\section{Change in amplitude and change in blood flow}

There was a correlation between change in amplitude and change in blood flow $(r=0.5477 ; p<0.05)$ (Fig. 5).

\section{DiscuSSION}

Measurement of ABI is a useful testing method for PAD diagnosis. ABI values $\leq 0.9$ have $95 \%$ sensitivity for the arteriographic detection of PAD. Specificity for angiographically documented PAD is $90 \%{ }^{15)}$ ABI measurement is an essential requirement for PAD screening. ${ }^{16,17)}$ Although devices are available for the simple calculation of ABI, the laser Doppler flowmeter used in this study has the advantages of being non-invasive and enabling continuous blood flow measurement. In our results, we found differences between the normal $\mathrm{ABI}$ and low ABI groups in the blood flow waveforms recorded. The amplitude of the waveform was significantly smaller in the low ABI group compared with the normal ABI group. The change in amplitude as a result of the imposition of leg extension and elevation stress was also smaller in the low ABI group. In addition, there was a positive correlation between change in amplitude size and $\mathrm{ABI}$, meaning that it may be possible to carry out screening for PAD by measuring the change in amplitude size before and during stress.

We found that the change in blood flow as a result of the imposition of leg extension and elevation stress was significantly lower in the low ABI group compared with the normal ABI group. This means that there is little variation in blood flow in patients with PAD despite the imposition of stress. When the cut-off point of blood flow change was $65 \%$, MILDF had a sensitivity of $81 \%$ and specificity of $58 \%$ in this study. A decrease in blood flow of more than $65 \%$ during leg elevation stress compared with blood flow at the rest (before stress) might indicate PAD. MILDF might be useful in the screening of PAD; however, the results in the present study did not consider the severity of PAD. Furthermore, a study with a large sample size is needed to evaluate whether MILDF could show the severity of PAD. In addition, there was a correlation between amplitude and change in blood flow. These results suggest that blood vessels have weak reactivity in cases of suspected PAD. ABI reflects the stenosis or occlusion of large blood vessels in the legs, and there has been insufficient investigation to say whether or not it reflects blood flow in peripheral tissue. Nevertheless, the positive correlation between change in blood flow and $A B I$ suggests the possibility that $A B I$ can be predicted from change in blood flow before and after leg 

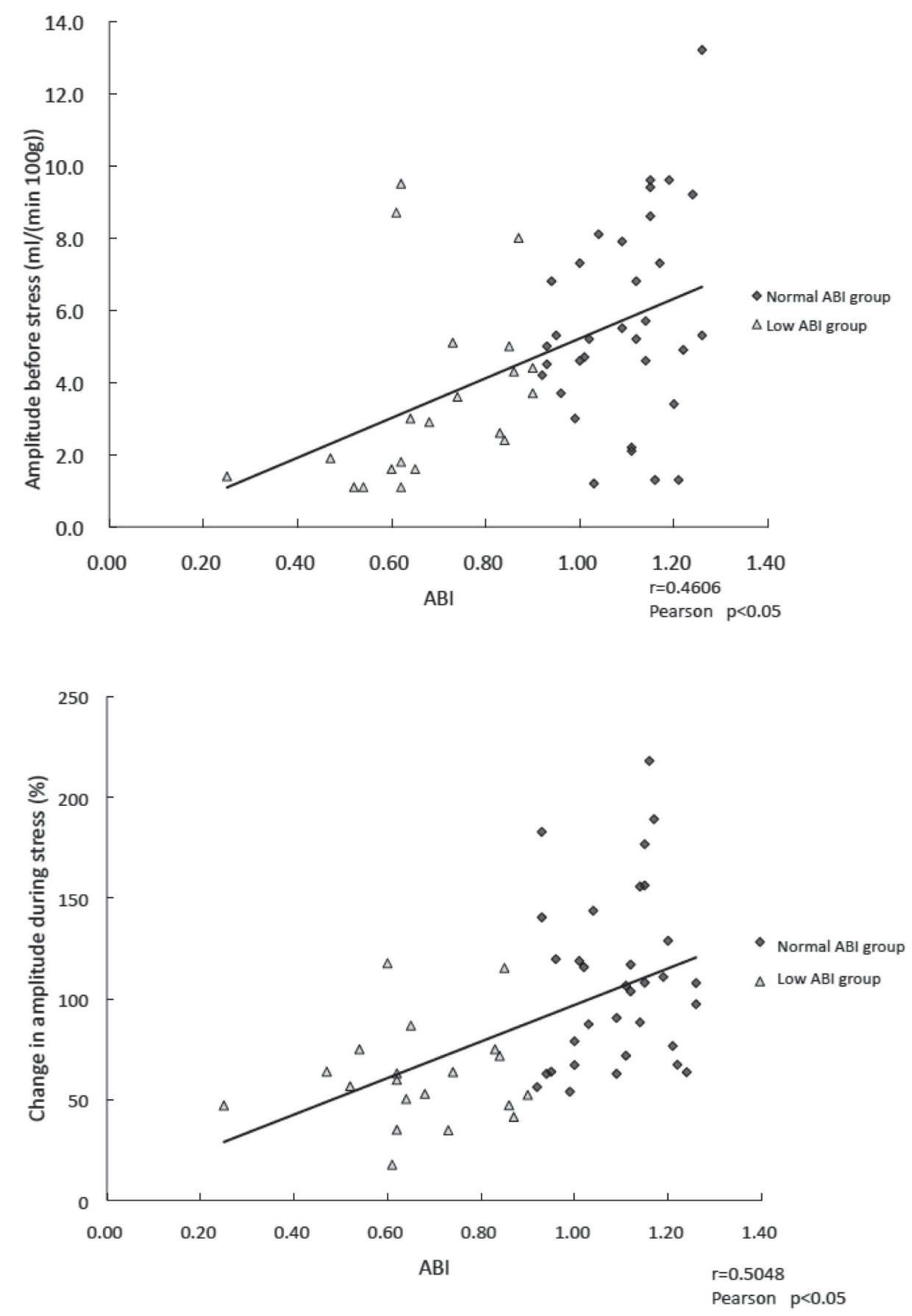

Fig. 3 Correlation between amplitude and ABI.

(a) Before stress

There was a correlation between amplitude and ABI before stress $(r=0.4606$; $p<0.05)$.

(b) During stress

There was a correlation between change in amplitude (\%) and ABI during stress $(r=0.5048 ; p<0.05)$.

ABI, ankle-brachial pressure index 


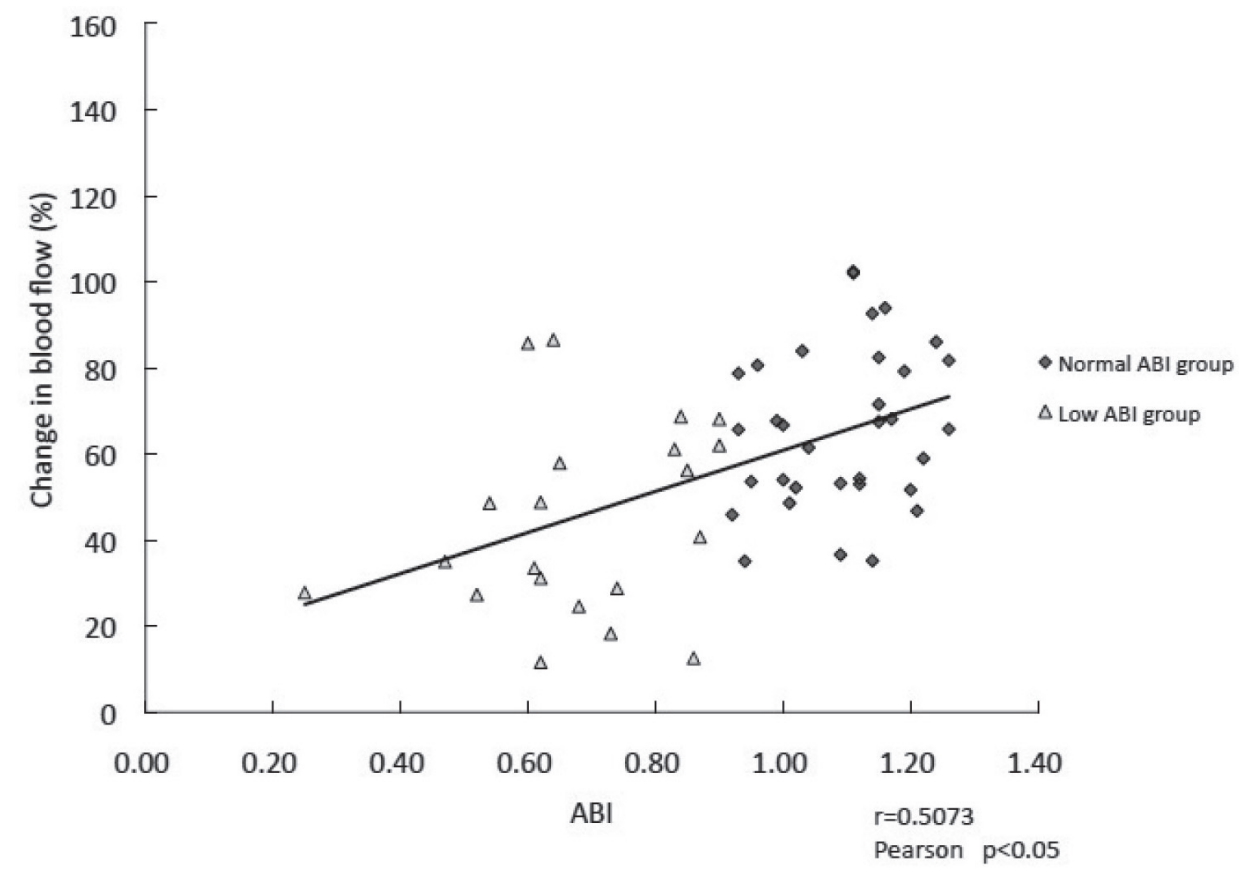

Fig. 4 Correlation between change in blood flow and ABI.

There was a correlation between change in blood flow and ABI $(r=0.5073 ; p<0.05)$.

$\mathrm{ABI}$, ankle-brachial pressure index

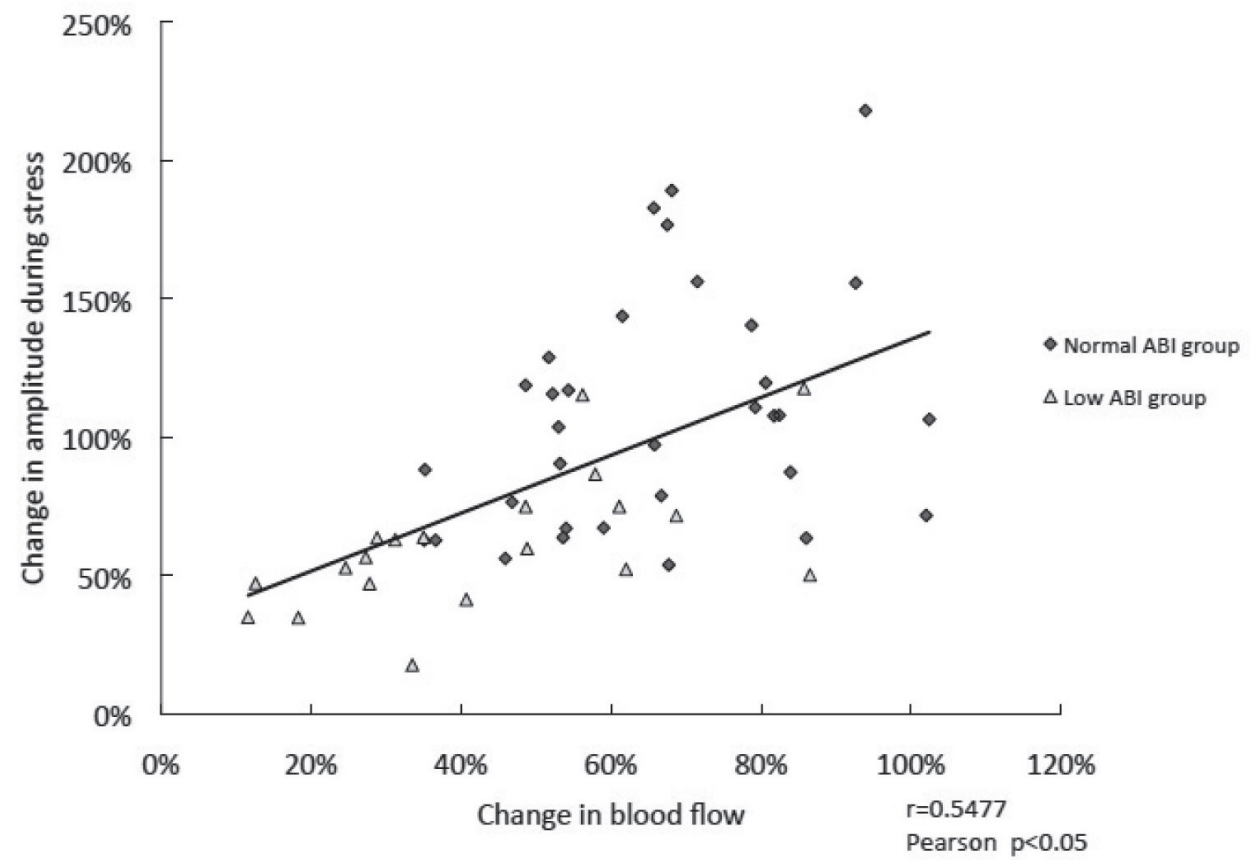

Fig. 5 Correlation between change in amplitude and change in blood flow.

There was a correlation between change in amplitude and change in blood flow $(r=0.5477 ; p<0.05)$. 
extension and elevation stress test. This study included only a small number of patients, however, and was limited by the fact that it was not possible to calculate the odds ratio for amplitude and change in blood flow that would constitute screening for PAD. If a large-scale study were carried out, this would enable the prediction of $\mathrm{ABI}$ from amplitude and change in blood flow. There are cases, however, in which it is not possible to obtain an accurate value for ABI because of a rise in ankle blood pressure due to diabetes, kidney failure, and vascular calcification. Cases which show false-positive ABI results should be examined using the TBI, which results in fewer false-positive findings compared with ABI. If TBI obtained by measuring blood pressure at the great toe is less than 0.7, then PAD is suspected. ${ }^{18,19)}$ In a comparison of TBI and ABI among patients who underwent examination in a specialist vascular outpatient clinic, both ABI and TBI were low in $34 \%$ of cases. ${ }^{20)}$ In $17 \%$ of cases, ABI was normal, and only TBI was low. ${ }^{20)}$ Because the MILDF used in this study measured peripheral blood flow in the great toe, it may also be applicable to screening for vascular lesions in patients with other vascular disorders causing peripheral stenotic lesions, diabetes, and kidney failure. The present study, however, did not investigate whether there was any correlation among TBI, amplitude, and change in blood flow. Further research is required to determine whether there is any correlation between TBI and values measured by LDF. Further research is also required to determine whether PAD screening is possible in patients with PAD and LSS, both of which have characteristic symptoms of intermittent claudication and similar ages of onset. LSS is complicated by PAD in $6.7 \%$ of cases, occurs mainly in elderly people, and has a high frequency of cardiovascular complications. ${ }^{21)}$ Whether screening is possible in patients with LSS complicated by PAD requires further study.

The MILDF used in this study has also been reported as a useful testing device for evaluating therapeutic efficacy in skin disorders. ${ }^{22)}$ It can also be anticipated to be of use as a tool for evaluating therapeutic efficacy in PAD patients. Further studies of blood flow waveform and changes in blood flow in PAD patients is required.

The MILDF used in this study can be used to screen for low ABI, by comparing amplitude and change in blood flow before and after leg extension and elevation stress. When the cut-off point of blood flow change using MILDF is $65 \%$, sensitivity for PAD is $81 \%$. Further study may enable LDF to be used as a simple PAD screening tool in outpatient clinics other than specialist vascular departments.

\section{REFERENCES}

1) Verbiest H. A radicular syndrome from developmental narrowing of the lumbar vertebral canal. J Bone Joint Surg Br 1954; 36: 230-7.

2) Verbiest H. Neurogenic intermittent claudication. Amsterdam: North-Holland, 1969: 79-150.

3) Criqui MH, Fronek A, Barrett-Connor E, Klauber MR, Gabriel S, Goodman D. The prevalence of peripheral arterial disease in a defined population. Circulation 1985; 71: 510-5.

4) Hiatt WR, Hoag S, Hamman RF. Effect of diagnostic criteria on the prevalence of peripheral arterial disease. The San Luis Valley Diabetes Study. Circulation 1995; 91: 1472-9.

5) Selvin E, Erlinger TP. Prevalence of and risk factors for peripheral arterial disease in the United States: results from the National Health and Nutrition Examination Survey, 1999-2000. Circulation 2004; 110: 738 43.

6) Resnick HE, Lindsay RS, McDermott MM, Devereux RB, Jones KL, Fabsitz RR, et al. Relationship of high and low ankle brachial index to all-cause and cardiovascular disease mortality: the Strong Heart Study. Circulation 2004; 109: 733-9.

7) Norgren L, Hiatt WR, Dormandy JA, Nehler MR, Harris KA, Fowkes FG, et al. Inter-society consensus for the management of peripheral arterial disease (TASC II). Eur J Vasc Endovasc Surg 2007; 33: S1-75.

8) Hirsch AT, Criqui MH, Treat-Jacobson D, Regensteiner JG, Creager MA, Olin JW, et al. Peripheral arterial disease detection, awareness, and treatment in primary care. JAMA 286; 1317-24.

9) Dhindsa M, Sommerlad SM, DeVan AE, Barnes JN, Sugawara J, Ley O, et al. Interrelationship amoung noninvasive measures of postischemic macro- and microvasucular reactivity. J Appl Physiol 2008; 105: 427-32.

10) Mahe G, Leftheriotis G, Picquet J, Jaquinandi V, Saumet JL, Abraham P. A normal penile pressure cannot rule out the presence of lesions on the arteries supplying the hypogastric circulation in patients with arterial claudication. Vasc Med 2009; 14: 331-8.

11) Higurashi $E$, Sawada R, Ito T. An integrated laser blood flowmeter. J Lightwave Technol 2003; 21: 591-5.

12) Kiyokura T, Mino, S, Shimada J. Wearable micro laser blood flowmeter. NTT Technical Journal 2005; 11: 24-7. (in Japanese).

13) Kiyokura T, Mino S, Shimada J. Wearable Blood Flowmeter: NTT Technical Review 2006; 4: 38-43. (in Japanese).

14) Hoshino S, Kikuchi S, Watanabe T, Sato K, Seino H, MIdorikawa H. Clinical guidebook for arteriosclerosis obliterans. Kita Media (Tokyo). 2002; 5-20. (in Japa- 
nese)

15) Guo X, Li J, Pang W, Zhao M, Luo Y, Sun Y, et al. Sensitivity and specificity of ankle-brachial index for detecting angiographic stenosis of peripheral arteries. Circ J 2008; 72: 605-10.

16) Ankle Brachial Index Collaboration, Fowkes FG, Murray GD, Butcher I, Heald CL, Lee RJ, et al. Ankle brachial index combined with Framingham Risk Score to predict cardiovascular events and mortality: a meta-analysis. JAMA 2008; 300: 197-208.

17) Criqui MH, Ninomiya JK, Wingard DL, Ji M, Fronek A. Progression of peripheral arterial disiease predicts cardiovascular disease morbidity and mortality. J Am Coll Cardiol 2008; 18: 1736-42.

18) Bird CE, Criqui MH, Fronek A, Denenberg JO, Klauber MR, Langer RD. Quantitative and qualitative progression of peripheral arterial disease by non-invasive testing. Vasc Med 1999; 4: 15-21.
19) Aboyans V, Criqui MH, Denenberg JO, Knoke JD, Ridker PM, Fronek A. Risk factors for progression of peripheral arterial disease in large and small vessels. Cirsulation 2006; 113: 2623-9.

20) Matsui K, Ikeda U, Murakami Y, Yoshioka T, Suzuki T, Shimada K. Analysis of measurement of TBI for diagnosis of PAD. J Jpn Coll Angiol 2003; 43: 536. (in Japanese)

21) Uesugi K, Sekiguchi M, Kikuchi S, Kanayama M, Takahashi K, Chiba K, et al. Lumbar spinal stenosis with peripheral disease. ISSLS 2010: GP141.

22) Kido M, Takeuchi S, Hayashida S, Urabe K, Sawada R, Furue M. Assessment of abnormal blood flow and efficacy of treatment in patients with systemic sclerosis using a newly developed microwireless laser Doppler flowmeter and arm-raising test. Br J Dermatol 2007; 157: 690-7. 